\title{
Author Correction: Realization of Lieb lattice in covalent-organic frameworks with tunable topology and magnetism
}

Bin Cui $\mathbb{D}$, Xingwen Zheng, Jianfeng Wang, Desheng Liu, Shijie Xie \& Bing Huang (i)

Correction to: Nature Communications https://doi.org/10.1038/s41467-019-13794-y, published online 2 January 2020.

The original version of this Article contained an error in the first sentence of the Acknowledgements, which incorrectly listed the funding number as ' 11574188 '. The correct version of the funding number is ' 11574118 '.

This has been corrected in both the PDF and HTML versions of the Article.

Published online: 29 January 2020

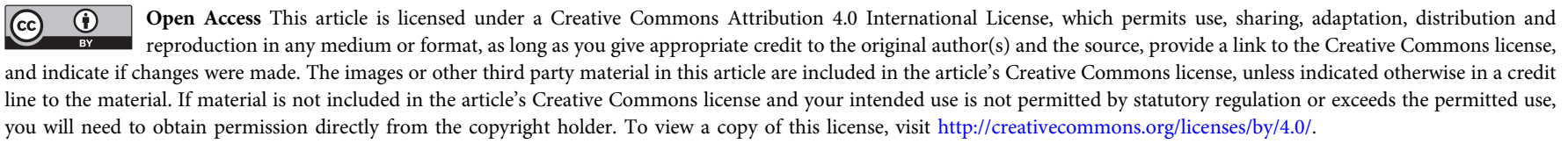

(C) The Author(s) 2020 